\title{
MACRÓFAGOS ALVEOLARES EN BOVINOS SOMETIDOS A HIPOXIA CRÓNICA
}

\author{
María E. Vásquez C. ${ }^{1}$, Milder Ayón S. ${ }^{1}$, Gilberto Santillán A. ${ }^{2}$, Raúl Rosadio A. ${ }^{3}$ y \\ Sergio Cueva M. ${ }^{1}$
}

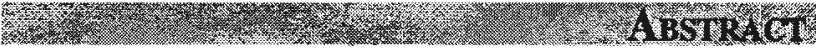

The impact of chronic hypoxia on alveolar macrophages in cattle

Alveolar macrophages (AM) play an important role in the regulation of local immune activity in the lung through production of bioactive factors in response to inflammatory processes and hypoxia. In the present study, the impact of hypoxia was examined by determining pulmonary AM and leucocyte numbers in lung lavage samples from 10 male Holsteins (aged 18-30 months) reared at high altitude and 9 reared at sea level. While total leukocyte counts for the high altitude $(216.62 \pm 106.22 / \mu \mathrm{l})$ and sea level $(117.09 \pm 15.71 /$ $\mu \mathrm{l})$ animals were not statistically different, mean $\mathrm{AM}$ values were $(\mathrm{p}<0.02)$ with greater values for the former $202.37 \pm 91.53 / \mu \mathrm{l}(95.5 \pm 2.5 \%)$ than for the latter group $75.75 \pm 17.84 / \mu \mathrm{l}$ $(65.11 \pm 11.62)$. Conversely, the number of lymphocytes was significantly higher $(\mathrm{p}<0.0004)$ at sea level $10.92 \pm 4.19 / \mu \mathrm{l}(8.83 \pm 2.63 \%)$ than at altitude $(0.62 \pm 0.35 \%)$, as were the values for polymorphonuclear cells $(\mathrm{PMN})(\mathrm{p}<0.0008)$ at $25.42 \pm 10.83 \%$ for sea level and $3.67 \pm 2.28 \%$ at high altitude. No differences in the frequency of basophils and eosinophils were found ( $p>0.05)$ at the two elevations. The increased numbers of alveolar AM at high elevation may possibly be a source of cytokines responsible for changes associated with chronic hypoxia at pulmonary level.
\end{abstract}

Key words: Alveolar macrophage (AM), hypoxia, cattle.

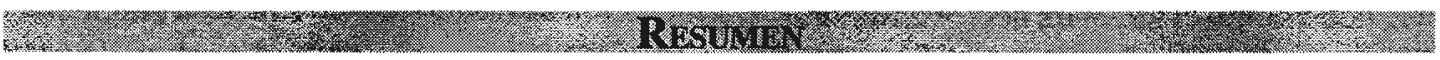

Los macrófagos alveolares (MA) juegan un rol importante en la regulación de la actividad inmune local en el pulmón, a través de la producción de factores bioactivos que son indispensables durante el daño pulmonar y la respuesta a procesos inflamatorios e hipoxia. El presente trabajo tuvo por objetivo determinar el número de MA y la cuenta leucocitaria. Para tal efecto se utilizaron 19 bovinos Holstein, machos, de 18 a 30 meses de edad, 10 nacidos y criados a 3320 m de altitud y 9 nacidos y criados a nivel del mar. El líquido recolectado del lavado broncoalveolar de pulmones extraídos después del sacrificio se utilizó para determinar la cuenta leucocitaria total en la cámara de Neubauer y para los frotices que fueron coloreados con May-Grünwald Giemsa, en los que se realizó la cuenta diferencial incluyendo MA. El número de los diferentes tipos de leucocitos por microlitro se obtuvo mediante un proceso matemático. El número de leucocitos totales de $216.62 \pm 106.22 / \mathrm{ml}$ en el grupo de altura fue mayor que $117.09 \pm 15.71 / \mathrm{ml}$ en el grupo de nivel del mar, sin embargo esta diferencia no fue significativa ( $p>0.05$ ). El valor promedio de MA en la altura de $202.37 \pm 91.53 / \mathrm{ml}$ $(95.5 \pm 2.50 \%)$ fue significativamente mayor $(\mathrm{p}<0.02)$ que $75.75 \pm 17.84 / \mathrm{ml}$ $(65.11 \pm 11.62 \%)$ a nivel del mar. El número de linfocitos de $10.92 \pm 4.19 / \mathrm{ml}(8.83 \pm 2.63 \%)$ en el grupo de nivel del mar fue significativamente mayor $(\mathrm{p}<0.0004)$ que $1.52 \pm 1.20 / \mathrm{ml}$

\footnotetext{
1 Laboratorio de Anatomía y Fisiología, FMV-UNMSM. E-mail: Elith_v@lycos.com

2 Laboratorio de Histopatología, FMV-UNMSM

${ }^{3}$ Laboratorio de Microbiologia y Virología, FMV-UNMSM
} 
en el grupo de nivel del mar fue significativamente mayor $(\mathrm{p}<0.0004)$ que $1.52 \pm 1.20 / \mathrm{ml}$ $(0.62 \pm 0.35 \%)$ del grupo de altura. El valor de polimorfonucleares en el grupo de nivel del mar de $25.42 \pm 10.83 \%$ fue significativamente mayor $(p<0.0008)$ que $3.67 \pm 2.28 \%$ en el grupo de altura. Los valores para basófilos y eosinófilos no fueron diferentes entre ambos grupos. Los resultados encontrados sugieren que la hipoxia podría estar activando a MA para la liberación de sustancias involucradas en el complejo proceso de muscularización vascular y desencadenando la hipertensión arterial pulmonar.

Palabras clave: Macrófago alveolar, hipoxia, bovinos, altura.

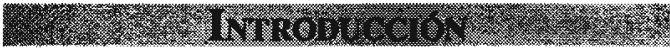

La falta de adaptación de las especies domésticas a las grandes alturas, lleva a la presentación de la enfermedad conocida como mal de altura, que es frecuente en bovinos y aves trasladados y criados en altitudes mayores a los $2150 \mathrm{msnm}$ (Peña et al., 1992). Bovinos transportados a grandes alturas desarrollan la enfermedad en un rango de 10 a 40\% (Will y Alexander, 1970). Muchos factores como prostaglandinas, leucotrienos, tromboxanos y citoquinas tienen acción en el desarrollo de la respuesta a hipoxia. En esta respuesta los macrófagos alveolares (MA), aparte de tener una actividad secretora de moléculas amplificadoras de la respuesta inmune, sintetizan y secretan sustancias como el factor de necrosis tumoral (TNF), interleuquina-1 (IL-1) y citoquinas quimiotácticas (Hempel et al., 1996). Hipoxia activa la producción del factor inductor de hipoxia-1 (HIF-1) (Yu et al., 1998) y respuesta de crecimiento temprano-1 (Egr1) en MA, endotelio vascular, músculo liso vascular y epitelio alveolar; teniendo éstos la capacidad de modular la expresión de genes que están involucrados en la remodelación de la matriz extracelular y del músculo liso en la respuesta pulmonar a hipoxia y en el desencadenamiento de la hipertensión arterial pulmonar (Yan et al., 2000), siendo mediadores esenciales en el mantenimiento de la homeostasis y del transporte de oxígeno celular (Semenza, 2000).

MA sometidos a hipoxia incrementan el bandeo de RNAm para TNF, IL-1 e IL1ra (Leeper-Woodford y Detmer, 1999), incrementándose la liberación de TNF $\alpha$, e
IL-1 $\beta$ (Ndengele et al., 2000). La activación del factor nuclear de IL-6 (NF-IL-6) resulta en un incremento de la expresión de IL-6, que es una citoquina con propiedades antiinflamatorias (Maeda et al., 1994), y éste puede ser el mecanismo limitante de la inflamación en casos de isquemia e hipoxia (Yan et al., 1995).

Se sabe que TNF $\alpha$ inicia la cascada de secreción de citoquinas regulando la expresión de genes importantes en la respuesta inflamatoria, también estimula la liberación de $I L-1 \beta$ que actúa como quimioatrayente causando adherencia leucocitaria y proliferación de células endoteliales (Gordon, 1995). Otro mecanismo que es activado por hipoxia es el incremento de la inducción del óxido nítrico sintetasa endotelial (eNOS) (Li et al., 1999), el cual es importante en la producción de óxido nítrico (ON), quién tiene funciones multifacéticas como activación de macrófagos, vasodilatación y neurotransmisión (NHLBI, 1999); y la producción de éste en las vías aéreas, es dependiente del nivel de oxígeno inspirado (Ide $e t$ al., 1999). La producción de citoquinas como IL-1 $\beta$, TNF $\alpha$ y proteína inflamatoria de macrófagos $1 \alpha$ (MIP-1 $\alpha$ ), decrecen la producción de ON en MA (Raychaudhuri et al., 1999). Estos cambios en la expresión del NOS han sido correlacionados con el proceso de remodelación vascular pulmonar en hipertensión inducida por hipoxia (Li et al., 1999).

Por tal motivo se planteó el presente trabajo con el fin de determinar el número de macrófagos alveolares en bovinos Holstein machos nacidos y criados en altura com- 
parados con bovinos de nivel del mar con la finalidad de entender la patogenia de la enfermedad conocida como mal de altura.

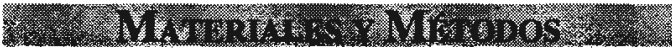

\section{Lugar de ejecución}

El presente trabajo se llevó a cabo en el Camal del distrito de Jauja, C.I.-IVITAEl Mantaro, Junín, y en el Camal Frigorífico José Olaya (Lima) y los Laboratorios de Fisiología y Anatomía Animal e Histopatología de la FMV-UNMSM-Lima.

\section{Animales}

Para el presente trabajo se utilizaron 19 bovinos machos de la raza Holstein con edades comprendidas entre 18 a 30 meses (10 nacidos y criados en altura y 9 a nivel del mar).

\section{Método Experimental}

Los animales fueron divididos en dos grupos: 10 animales de altura (Camal del distrito de Jauja) y 9 animales a nivel del mar (Camal José Olaya- Lima), en los cuales se realizó el lavado broncoalveolar según las técnicas descritas por Larson y Busch (1985) y Sweeney et al. (1992). Se realizó un corte a unos $7 \mathrm{~cm}$ de la bifurcación de la tráquea, por el cual se introdujo un catéter de $0.8 \mathrm{~cm}$ de diámetro por $50 \mathrm{~cm}$ de largo, se dirigió por el bronquio primario hasta la porción terminal, primero del lado izquierdo. Se depositó $30 \mathrm{ml}$ de cloruro de sodio al $0.09 \%$, para luego retirarse por succión suave, el líquido recolectado se depositó en tubos graduados heparinizados. Lo mismo se repitió con el lado derecho. Las muestras se transportaron al laboratorio en un envase térmico con hielo para su procesamiento. En el laboratorio se midió la cantidad de líquido recolectado de cada pulmón, determinándose el porcentaje del mismo. Luego se reali- zó la cuenta leucocitaria en la cámara de Neubauer, según la técnica descrita por Benjamin (1991).

Para encontrar el número de leucocitos por microlitro se utilizó la siguiente fórmula:

$$
\begin{aligned}
& \left(\mathrm{V}_{\text {der }} \times \mathrm{NCC}_{\mathrm{der}}\right)+\left(\mathrm{V}_{\mathrm{izq}} \times \mathrm{NCC}_{\mathrm{izq}}\right) \\
& \text { No. } \mathrm{Cel} / \mu \mathrm{l}=\frac{\left(\mathrm{V}_{\mathrm{der}} \times \mathrm{N}_{\mathrm{der}}+\mathrm{V}_{\mathrm{izq}}\right)}{\left(\mathrm{ders}_{\mathrm{g}}\right)+\left(\mathrm{V}_{\mathrm{iqq}} \times \mathrm{CC}_{\mathrm{zq}}\right)} \\
& \mathrm{V}_{\mathrm{der}}: \quad \text { Volumen recolectado del } \\
& \text { pulmón derecho } \\
& \mathrm{V}_{\mathrm{izq}} \text { : Volumen recolectado del } \\
& \text { pulmón izquierdo } \\
& \mathrm{NCC}_{\text {der }} \text { : Número de células contadas } \\
& \text { del lado derecho } \\
& \mathrm{NCC}_{\text {izq }} \text { : Número de células contadas } \\
& \text { del lado izquierdo }
\end{aligned}
$$

Las muestras fueron centrifugadas a 900 revoluciones por minuto, durante $5 \mathrm{mi}-$ nutos, del precipitado se hicieron extensiones en dos láminas por muestra. Las muestras fueron fijadas en alcohol de $96^{\circ}$ y coloreadas con la tinción de May-GrünwaldGiemsa. Se hizo una cuenta diferencial con 200 células por lámina, obteniéndose los porcentajes de MA, polimorfonucleares, linfocitos, basófilos y eosinófilos. Luego por proceso matemático se obtuvo el número de los diferentes tipos celulares por microlitro.

Después del lavado broncoalveolar, se tomaron muestras del parénquima pulmonar para histopatología. Las cuales fueron procesadas según las técnicas de preparación de láminas histológicas y fueron coloreadas con Hematoxilina-Eosina. En estas láminas se buscó alteraciones patológicas.

\section{Análisis estadístico}

Los resultados fueron analizados mediante análisis de varianza de una sola vía, con un $95 \%$ de nivel de confianza, a fin de hallar las diferencias entre ambos grupos. 


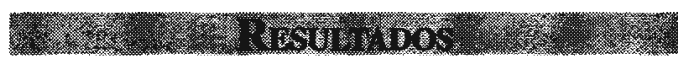

El líquido recolectado fue similar en ambos grupos ( $\mathrm{p}>0.05$ ) (Cuadro 1). Se observó mayor número de leucocitos $/ \mathrm{ml}$ en bovinos de altura $216.62 \pm 106.22 / \mu \mathrm{l}$ que en bovinos a nivel del mar $117.09 \pm 15.71 /$ $\mu \mathrm{l}$, no siendo diferentes ( $\mathrm{p}>0.05$ ). Se obtuvo un mayor número de $\mathrm{MA}(\mathrm{p}<0.02)$ en bovinos de altura $202.37 \pm 91.53 / \mu l$ que a nivel del mar 75.75 $\pm 17.84 / \mu l$. Los linfocitos fueron mayores $(\mathrm{p}<0.0004)$ en bovinos a nivel de mar $10.92 \pm 4.19 / \mu \mathrm{l}$ que en el grupo de altura $1.52 \pm 1.20 / \mu \mathrm{l}$. Polimorfonucleares, eosinófilos y basófilos no fueron diferentes ( $p>0.05$ ) entre ambos grupos (Cuadro 2).

La cuenta diferencial mostró un mayor $(\mathrm{p}<0.0001)$ porcentaje de MA $95.5 \pm$ $2.50 \%$ en bovinos de altura, que en bovinos a nivel del mar $65.11 \pm 11.62 \%$. Polimorfonucleares y linfocitos fueron mayores en animales a nivel del mar $(p<0.0000)$, que en animales de altura. Eosinófilos y basófilos no fueron diferentes ( $\mathrm{p}>0.05$ ) (Cuadro 3).

\section{Descripción de láminas de histopatología:}

Las láminas de las muestras de animales de altura presentan ligero engrosamiento de los septos interalveolares, engrosamiento moderado de las capas musculares de los vasos sanguíneos. En algunos de ellos hay ligero engrosamiento de la pleura, así como infiltración de tejido conectivo cerca de los vasos sanguíneos; observándose en algunos animales infiltración focal de mononucleares. Las láminas de los animales de nivel de mar, presentan de ligero a moderado engrosamiento de los septos interalveolares, algunos animales presentaron de ligero a moderado engrosamiento de la pleura, moderada infiltración focal de mononucleares y focos de infiltración leucocitaria mixta.

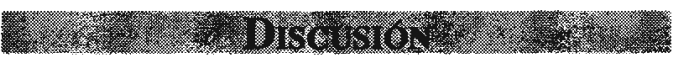

Como se observa en el Cuadro 2, el número de leucocitos totales fue ligeramente mayor en altura que a nivel del mar, no llegando a tener diferencias estadísticas; pero el porcentaje de macrófagos alveolares (MA) así como el número de los mismos por $\mu \mathrm{l}$ fue mayor $(p<0.02)$ en el grupo de bovinos de altura, estos resultados coinciden con lo encontrado por Sulkowska (1997), quienes señalan que hay un incremento de MA en animales sometidos a condiciones de hipoxia hipobárica experimental de extrema altura (5500 m de altitud). Karakurum et al. (1994) y Doerschuk et al. (1990) señalan que hay quimiotaxis leucocitaria y leucostasis tisular, y esto se ve incrementado por un alto secuestro de monocitos circulantes debido a la baja presión en los capilares pulmonares. $\mathrm{Si}$ bien en el presente trabajo los animales no fueron sometidos a condiciones hipobáricas de extrema altura, ellos han tenido una respuesta similar a los resultados obtenidos por estos investigadores.

En animales a nivel del mar el número de $\mathrm{MA} / \mu \mathrm{l}$ fue de $75.75 \pm 17.84$, similar a lo encontrado por Weissbecker et al. (1969) y Arend y Mannik (1973), de 44 y $90 \mathrm{MA} /$ $\mu l$ respectivamente. En animales de altura los $\mathrm{MA} / \mu \mathrm{l}$ fue de $202.37 \pm 91.53$ mayor a lo reportado por estos investigadores. En el presente trabajo, en animales a nivel del mar los $\mathrm{MA} / \mu \mathrm{l} 75.75 \pm 17.84$, fue menor a lo encontrado por Lay et al. (1986), quienes reportaron $85.7 \pm 8.7 \%$ en animales normales, cabe destacar que este porcentaje fue menor a los animales de altura, $95.5 \pm 2.5 \%$, del presente trabajo. Los valores en porcentajes mencionados varían dependiendo de las condiciones en que se encuentran los animales, edad de los mismos y las técnicas usadas para la recolección de muestras. Se postula que por la edad de los animales, ambos grupos fueron sometidos alguna vez a condiciones medioambientales y de manejo adversas, lo cual podrían estar influenciando a algunos de los resultados. 
Cuadro 1. Volumen suministrado(VS) y volumen recolectado (VR) en lavado broncoalveolar de bovinos Holstein machos de altura y a nivel del mar.

\begin{tabular}{lcccc}
\hline \multicolumn{1}{c}{ NIVEL } & $\mathrm{N}$ & $\begin{array}{c}\text { VS } \\
(\mathrm{ml})\end{array}$ & $\begin{array}{c}\text { VR }(\mathrm{ml}) \\
\mathrm{X} \pm \mathrm{NC}\end{array}$ & $\begin{array}{c}\% \\
\mathrm{X} \pm \mathrm{NC}\end{array}$ \\
\hline \multirow{2}{*}{ Altura } & 10 & 60.0 & $23.61 \pm 4.63$ & $39.34 \pm 11.72$ \\
Nivel del mar & 9 & 60.0 & $20.33 \pm 3.31$ & $33.89 \pm 5.51$ \\
\hline $\mathrm{n} \quad$ Número de animales & & & & \\
$\mathrm{X}: \quad \begin{array}{l}\text { Promedio } \\
\mathrm{NC}: \text { Nivel de confianza }\end{array}$ & & & &
\end{tabular}

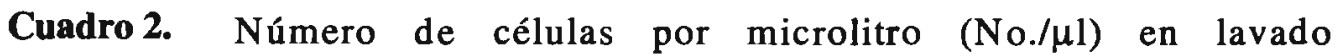
broncoalveolar de bovinos Holstein machos de altura y a nivel del mar.

\begin{tabular}{lcc}
\hline \multicolumn{1}{c}{ TIPO CELULAR } & ALTURA & NIVEL DEL MAR \\
\hline Número de animales & $\mathrm{X} \pm \mathrm{NC}$ & $\mathrm{X} \pm \mathrm{NC}$ \\
Leucocitos totales & $216.62 \pm 106.22$ & $117.09 \pm 15.71$ \\
MA & $202.37 \pm 91.53$ & $75.75 \pm 17.84^{*}$ \\
Polimorfonucleares & $12.44 \pm 15.17$ & $29.72 \pm 14.26$ \\
Linfocitos & $1.52 \pm 1.20$ & $10.92 \pm 4.19^{*}$ \\
Eosinófilos & $0.25 \pm 0.30$ & $0.59 \pm 0.32$ \\
Basófilos & $0.04 \pm 0.07$ & $0.11 \pm 0.21$ \\
\hline $\mathrm{X}$ Promedio & & \\
NC: Nivel de confianza & & \\
$*: \quad$ Diferencia significativa & &
\end{tabular}

Cuadro 3. Cuenta diferencial (\%) de leucocitos en lavado broncoalveolar de bovinos Holstein machos en altura y a nivel del mar.

\begin{tabular}{lcc}
\hline \multicolumn{1}{c}{ TIPO CELULAR } & ALTURA & NIVEL DEL MAR \\
\hline Número de animales & $\mathrm{X} \pm \mathrm{NC}$ & $\mathrm{X} \pm \mathrm{NC}$ \\
MA & 10 & 9 \\
Polimorfonucleares & $95.50 \pm 2.50$ & $65.11 \pm 11.62^{*}$ \\
Linfocitos & $3.67 \pm 2.28$ & $25.42 \pm 10.83^{*}$ \\
Eosinófilos & $0.62 \pm 0.35$ & $8.83 \pm 2.63^{*}$ \\
Basófilos & $0.18 \pm 0.20$ & $0.56 \pm 0.30$ \\
\hline $\mathrm{X}: \quad$ Promedio & $0.03 \pm 0.05$ & $0.08 \pm 0.16$ \\
NC: Nivel de confianza & & \\
$*$ *: Diferencia significativa & &
\end{tabular}


Los animales nacidos y criados a nivel del mar, son más susceptibles a contraer enfermedades bacterianas determinado por las condiciones medioambientales en las cuales fueron criados. La respuesta al medioambiente se pudo observar y ha sido descrito al leer las láminas de histopatología. Los resultados encontrados con respecto a polimorfonucleares y linfocitos se puede entender como una respuesta normal de los individuos a su medio ambiente ya que ambos tipos celulares, son los primeros en responder y llegar al lugar de la inflamación (Tizard, 1998); allí ejercen las funciones de fagocitosis, destruyen sustancias exógenas $\mathrm{y}$ producen sustancias vasoactivas $\mathrm{y}$ quimiotácticas (Strom y Thomsen, 1990).

Los MA son la fuente primaria de producción de citoquinas inflamatorias en el pulmón y éstos pueden ser activados por una serie de condiciones (Czermak et al., 1999). En enfermedades pulmonares se incrementa la inducción y producción de $I L-1 b, I L-6$ y TNF $\alpha$ por la vía de NF-kappa $\beta$ (Drumm et al., 1998; Hulte et al., 1998), sucediendo lo mismo en casos de hipoxia (LeeperWoodford y Detmer, 1999; Ndengele et al., 2000). TNF $\alpha$ inicia la cascada de secreción de citoquinas regulando la expresión de genes (Gordon, 1995; Gordon et al., 1995), además TNF $\alpha$ actúa como activador autocrino para la generación de quimiocinas CXC y CC (Czermak et al. 1999)

Además la activación de MA para la producción de ON en casos de hipoxia es otro de los mecanismos involucrados en hipoxia y al mismo tiempo TNF $\alpha, I L-1 \beta$ y MIP- $1 \alpha$ secretado por MA disminuyen la producción de ON (Raychaudhuri et al., 1999; NHLBI, 1999; Raychaudhuri et al., 1999; Ide et al., 1999).

La hipoxia induce en los MA respuestas adaptativas complejas como la generación de Egr-1, HIF-1, que intervienen como mecanismos esenciales en la modulación de la homeostasis pulmonar en hipoxia (Semenza, 2000; Yan et al., 2000). Estos mecanis- mos de respuesta podrían estar involucrados en el aumento del número de macrófagos alveolares en bovinos sometidos a hipoxia ambiental crónica observado en el presente experimento.

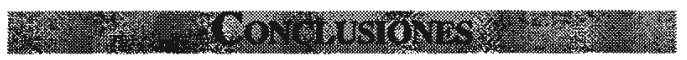

Bovinos Holstein machos nacidos y criados en altura presentan un mayor porcentaje y número de macrófagos alveolares/ $\boldsymbol{\mu l}$ determinado por lavado broncoalveolar que bovinos nacidos y criados a nivel del mar.

No se encontró variación en el número de leucocitos totales entre los grupos de altura y nivel del mar.

Se encontró mayor número y porcentaje de linfocitos en el grupo de nivel del mar que en el grupo de altura, polimorfonucleares sólo fue mayor en porcentaje en el grupo de nivel del mar; mientras que basófilos y eosinófilos no presentaron variación entre ambos grupos.

El mayor número de macrófagos alveolares encontrados en altura, sugiere que hipoxia podría estar activando a macrófagos alveolares para la liberación de sustancias involucradas en el complejo proceso de muscularización vascular y desencadenar hipertensión arterial pulmonar.

1. Arend, W. P. y M. Mannik. 1973. The macrophage receptor for IgG: Number and affinity of binding sites. J Immunol; 10:1455-1463.

2. Benjamin, M. M.. 1991. "Recuento de células sanguíneas". En: Manual de Patología Clínica en Veterinaria. Noriega Editores. 1a. ed, p:61-66. Ed. Limusa S.A. de C.V., México. 
3. Czermak, B. J., V. Sarma, N. M. Bless, H. Schmal, H. P. Friedl y P. A. Ward. 1999. In vitro and in vivo dependency of chemokine generation on $\mathrm{C} 5 \mathrm{a}$ and TNF-alpha. J Immunol; 162(4):23212325.

4. Doerschuk, C. M., G. P. Downe, D. E. Dohery, D. English y R. P. Gie. 1990. Leukocyte and platelet margination within the microvasculature of rabbit lung. J Appl Physiol; 68:1956-1961.

5. Drumm, K., R. Oettinger, R. Smolarski, M. Bay y K. Kienast. 1998. In vitro study of human alveolar macrophages inflamatory mediator transcriptions and releases induced by soot FR 101, Printex 90, titandioxide and chrysotile B. Eur J Med Res; 3(9):432-438.

6. Gordon, S. 1995. My favourite cell: The macrophage. Bioessays; 17:977-986.

7. Gordon, S., S. Clarke, D. R. Greaves y A. Doyle. 1995. Molecular Immunobiology of macrophages: Recent Progress. Current Opinions in Immunology; $7: 24-33$

8. Hempel, S. L., M. M. Monick y G. W. Hunninghake. 1996. Effect of hypoxia on release of $\mathrm{IL}-1$ and TNF by human alveolar macrophages. Am J Respir Cell Mol Biol; 14:170-176.

9. Hulte, L. M., H. Lindmark, H. Schersten, O. Wi Klund, F. N. Nilsson $y$ G. C. Riise. 1998. Butylated hydroxytoluene and $\mathrm{N}$-acetyleysteine attenuates tumor necrosis factor-alpha (TNF-a) secretion and TNF-a mRNA espression in alveolar macrophages from human lung transplant recipients in vitro. Transplatation; 66(3):364-369.

10. Ide, H., H. Nakano, T. Ogasa, S. Osanai, K. Kikuchi y J. Iwamoto. 1999. Regulation of pulmonary circulation by alveolar oxygen tension via airway nitric oxide. J Appl Physiol; 87(5):16291636.
11. Karakurum, M., R. Shreeniwas, J. Chen, D. Pinsky, S. D. Yan, M. Anderson, K. Sunouchi, J. Major, T. Hamilton y K. Kuwabara. 1994. Hypoxic induction of interleukin- 8 gene expression in human endothelial cells. J Clin Invest; 93:1564-1570.

12. Larson, V. L. y R. H. Busch. 1985. Equine tracheobronchial lavage: Comparison of lavage cytologic and pulmonary histopathologic findings. Am J Vet Res; 46(1): 144-146.

13. Lay, J. C., D. O. Slauson y W. L. Castleman. 1986. Volume-controlled bronchopulmonary lavage of normal and pneumonic calves. Vet Pathol; 23:673680.

14. Leeper-Woodford, S. K. y K. Detmer. 1999. Acute hypoxia increases alveolar macrophage tumor necrosis factor activity and alters NF-B expression. AJP: Lung Cell Mol Physiol; 276(6):L909L916.

15. Li, D., N. Zhou y R. A. Jhons. 1999. Soluble guanylate cyclase gene expression and localization in rat lung after exposure to hypoxia. AJP: Lung Cell Mol Physiol; 277(4):L841-L847.

16. Maeda, Y., M. Matsumoto, O. Hori, K. Kuwabara, S. Ogawa, S. D. Yan, T. Ohtsuki, T. Kinoshita, T. Kamada y D. M. Stern. 1994. Hypoxia/reoxygenationmediated induction of astrocyte interleukin 6: A paracrine mechanism potentially enhancing neuron survival. J Exp Med; 180:2297-2308 .

17. National Heart: Lung and Blood Institute (NHLBI). 1999. Role of Nitric oxide in the pathogenesis of lung disease. Protocol N ${ }^{\circ}$ 94-H-0141.

18. Ndengele, M.M., C.J. Bellone, A.J. Lechner y G.M. Matuschak. 2000. Brief hypoxia differentially regulates LPSinduced IL-1 and TNF-gene transcription in RAW 264.7 cells. Lung Cell and Mol Physiol; 278(6):L1289-L1296. 
19. Peña, J., A. S. de Aluja y R. Navarro. 1992. Insuficiencia Cardiaca congestiva derecha en becerras Holstein de reemplazo II. Clínica y Patología. Vet. Max., XXIII:2.

20. Raychaudhuri, B., R. Dweik, M. J. Connors, L. Buhrow, A. Malur, J. Drazba, A. C. Arroliga, S. C. Erzurum, M. S. Kavuru y M. J. Thomassen. 1999. Nitric oxide blocks nuclear factor-kappa $B$ activation in alveolar macrophages: Am J Resp Cell Mol Biol; 21(3):311316.

21. Semenza, G.L. 2000. HIF-1: mediator of physiological and pathophysiological responses to hypoxia. Am J. Physiol; 88(4):1474-1480.

22. Strom, H. y H. K. Thomsen. 1990. Effects of proinflammatory mediators on canine neutrophil chemotaxis and aggregation. Vet. Immunol Immunopathol; 25:209-218.

23. Sulkowska, M. 1997. Morphological studies of the lungs in chronic hypobaric hypoxia. Pol J Pathol; 48(4):225-234.

24. Sweeney, C. R., Y. Rossier, E. L. Ziener y S. R. Lindborg. 1992. Effects of lung site and fluid volume on results of bronchoalveolar lavage fluid analysis in horses. Am J Vet Res; 53(8):1376-1379.
25. Tizard; I. R. 1998. Inflamación. En: Inmunología Veterinaria, Cap. 5. la. ed, p: 47-60. Ed. McGraw-Hill Interamericana, México.

26. Weissbecker, L, R. D. Carpenter y P. C. Luchsinger. 1969. In vitro alveolar macrophage viability. Arch Environ Health; 18:256-259.

27. Will, D. H. y A. F. Alexander. 1970. High mountain (brisket) disease. Am Vet. Publ; 3:412-430.

28. Yan, S. F., I. Tritto, D. Pinsky, H. Liao, J. Huang, G. Fuller, J. Brett, L. May y D. Stern. 1995. Induction of interleukin $6(\mathrm{IL}-6)$ by hypoxia in vascular cells. Central role of the binding site for nuclear factor IL-6. J Biol Chem; 270: 11463-11471.

29. Yan, S., J. Lu, L. Xu, Y. S. Zou, J. Tongers, W. Kisiel, N. Mackman, D.J. Pinsky y D. M. Stern. 2000. Pulmonary expression of early growth response 1 : biphasic time course and effect of oxygen concentration. J Appl Physiol; 7:A 175-A180.

30. Yu, A. Y., M. G. Frid, LA. Shimoda, C. M. Wiener, K. Stenmark y G. L. Semenza. 1998. Temporal, spatial and oxygen-regulated expression of hypoxia-inducible factor-1 in the lung. Am J. Physiol; 275:L818-L826. 